liefert für Anthracen und Naphthalin:

$$
\begin{aligned}
\Gamma \chi_{n} & =\mathrm{B}_{1 u} \text { für } \tau_{x}, \\
& =\mathrm{A}_{u} \text { für } \tau_{y}, \\
& =\mathrm{B}_{3 u} \text { für } \tau_{z} .
\end{aligned}
$$

$\mathrm{A}_{u}$ und $\mathrm{B}_{1 u}$ sind nicht planare Molekülschwingungen. $\mathrm{B}_{3 u}$ ist eine planare Molekülschwingung ${ }^{31,32}$. Mit den Meßergebnissen [(Gl. (15)] folgt also, daß der Zerfall überwiegend über eine Gesamtheit von Molekülschwingungen der Symmetrie $\mathrm{B}_{1 u}$ und $\mathrm{A}_{u}$ erfolgt. Dieses Ergebnis stimmt ausgezeichnet mit den Berechnungen von Henry und Siebrand 12 überein. Ihre theoretische Arbeit zum Isotopieeffekt auf die Triplettlebensdauer teilweise deuterierter Naphthalinmoleküle begründet sich auf dem

31 H. Luther u. H.-J. Drewits, Z. Elektrochem. 66, 546 [1962].
Mechanismus des „Vibronic Spin-Orbit Coupling“ unter Verwendung von C-H nichtplanaren Schwingungen der Symmetrie $\mathrm{A}_{u}$ und $\mathrm{B}_{1}$ für $\tau_{y}$ und $\tau_{x}$. Wie bei der Bevölkerung der Naphthalintriplettzustände ${ }^{3}$ sind auch beim Zerfall nichtplanare Schwingungen beteiligt. Die ISC-Prozesse unterscheiden sich damit von den Prozessen der Inneren Konversion, bei denen nur planare Schwingungen einen Beitrag zur Energierelaxation liefern ${ }^{32}$.

Herrn Professor Dr. H. C. Wolf danken wir für die hervorragenden Arbeitsmöglichkeiten im 3. Physikalischen Institut und für die Förderung dieser Arbeit. Herrn Dr. N. KaRL, Herrn Dr. K. W. Benz (Stuttgart) und Herrn H. Zimmermann (Heidelberg) danken wir für die Herstellung der Kristalle. Die Arbeit wurde von der Deutschen Forschungsgemeinschaft mit Sachmitteln unterstützt.

32 U. Sommer, Dissertation Stuttgart 1969.

\title{
Resonanz-Raman-Effekt von Thiocyanatkomplexen einiger Übergangsmetalle
}

\author{
W. KrasSER und H. W. NÜRNBERG \\ Zentrallabor für Chemische Analyse der Kernforschungsanlage Jülich GmbH \\ (Z. Naturforsch. 25 a, 1394-1401 [1970] ; eingegangen am 25. Juli 1970)
}

\begin{abstract}
The thiocyanates of the transition metals iron, cobalt, copper as well as of rhenium and of technetium appear in solution as strongly coloured complexes. The resonance raman bands in the solvent acetonitrile are investigated. To achieve an unambiguous identification the infrared spectra were recorded too. The change in position and structure of the acetonitrile bands indicates strong complexation of iron, cobalt and copper with acetonitrile, thus indicating the existence of mixed acetonitrile-thiocyanate complexes. The resonance raman spectra of the rhenium- and technetiumthiocyanates present as tetramethyl ammonium salts show however no raman- and infrared-bands of complexed acetonitrile molecules.

In the raman spectrum of the thiocyanates of iron, cobalt and copper mainly the totally symmetric $\mathrm{C} \equiv \mathrm{N}, \mathrm{S}-\mathrm{C}, \mathrm{Me}-\mathrm{S}$ and $\mathrm{Me}-\mathrm{N}$ valence vibrations are observed, among which the $\mathrm{S}-\mathrm{C}$ vibration shows a remarkably high intensity. Besides, a series of bands is obtained which is interpreted partly as caused by decay products, and partly as bands of complexed acetonitrile. The thiocyanates of rhenium and of technetium show the three possible valence vibrations only. The high frequency of the $\mathrm{S}-\mathrm{C}$ valence indicates the N-coordination of the thiocyanate group.
\end{abstract}

\section{Einleitung und theoretische Aspekte}

Farblose, im UV absorbierende Stoffe liefern gewöhnlich normale Raman-Spektren. Erst die Einführung des Lasers als Erregerlichtquelle hat der

Sonderdruckanforderungen an Dr. W. KRASSER, Zentrallabor für Chem. Analyse der Kernforschungsanlage Jülich GmbH, D-5170 Jülich 1, Postfach 365.

1 W. Hayden Smith u. G. E. Leror, J. Chem. Phys. 45, 1778 [1966]. - B. Schrader u. M. Stockburger, Z. Anal. Chem. 216, 117 [1966]. - T. E. HaAs u. J. R. Hall, Spectrochim. Acta 22 A, 988 [1966]. - P. J. Hendra, Nature London 212, 179 [1966].
Raman-Spektroskopie den Zugang zu gefärbten Substanzen geöffnet. Untersuchungen verschiedener Autoren ${ }^{1-3}$ demonstrierten in den letzten 4 Jahren die großen Möglichkeiten, die sich durch den Einsatz des Lasers für die Raman-Spektroskopie ge-

2 P. J. Hendra u. P. M. Stratton, Chem. Rev. 69, 325 [1969].

3 Jahresbericht der Kernforschungsanlage Jülich $\mathrm{GmbH}$, 1966, 156. - W. Krasser u. H. W. Nürnberg, Naturwiss. 54, 134 [1967]. 
färbter anorganischer Verbindungen und Komplexe im kristallinen Zustand und auch in gelöster Phase ergeben (vgl. die Übersicht in Anm. ${ }^{4}$ ) .

Bei einer Reihe gefärbter anorganischer Komplexe tritt in gelöster Phase das interessante Phänomen des Resonanz-Raman-Effektes auf. Dieser von uns $^{3}$ erstmalig 1966 für anorganische Systeme an einigen gefärbten Komplexen in verschiedenen Solventien experimentell nachgewiesene Effekt wurde für verschiedene Thiocyanatkomplexe eingehender studiert. Bei gefärbten Substanzen mit Absorptionsbanden im sichtbaren Spektralbereich kann man generell folgende Fälle unterscheiden:

1. Bei relativ weiter Entfernung der Absorptionsbande von der Erregerlinie $v_{0}$ des Lasers erhält man ein normales und vollständiges Raman-Spektrum mit Ober- und Kombinationsschwingungen. Liegen Laserlinie und Absorptionsbande nahe beieinander, so bestehen folgende Möglichkeiten:

2. Es entsteht ein schwaches bzw. gar kein RamanSpektrum, da die Absorption dominierend bleibt.

3. Zeigt das Raman-Spektrum außergewöhnlich hohe Intensität, ist es unvollständig und enthält es im wesentlichen nur die totalsymmetrischen Schwingungen, so liegt ein Resonanz-Raman-Effekt vor.

Die Möglichkeit des Auftretens sehr intensiver Raman-Frequenzen bei gefärbten Substanzen, die eventuell bis zu der außerordentlichen Intensitätssteigerung eines Resonanzeffektes führt, ergibt sich unmittelbar aus Gl. (1) für den Polarisierbarkeitstensor ${ }^{\mathbf{5}, 6}$ :

$\alpha_{m n}^{\rho \sigma}=\frac{1}{h} \sum_{r} \frac{\left(M_{Q}\right)_{r n}\left(M_{\sigma}\right)_{n r}}{v_{r n}-v_{0}}+\frac{\left(M_{Q}\right)_{m r}\left(M_{\sigma}\right)_{r m}}{v_{r m}+v_{0}}$.

Die Intensität einer zu dem molekularen Übergang $m \rightarrow n$ gehörenden Raman-Linie ist im wesentlichen proportional dem Quadrat des Polarisierbarkeitstensors.

$h$ ist das Plancksche Wirkungsquantum. Für die Übergangsmomente gilt

$$
M_{n r}=\int_{-\infty}^{+\infty} \psi_{n}^{*} \boldsymbol{M} \psi_{r} \mathrm{~d} \tau \quad \text { etc. }
$$

${ }^{4}$ K. Schwochau u. W. Krasser, Z. Naturforsch. 24 a, 403 [1969]. - W. KRASSER, Naturwiss. 56, 213 [1969]. W. Krasser, Z. Naturforsch. 24 a, 1667 [1969]. - W. Krasser u. K. Schwochau, Z. Naturforsch. 25 a, 206 [1970]. - W. Krasser u. H. W. NÜrnberG, Spectrochim. Acta 26 A, 1059 [1970]. - W. Krasser, Ber. Bunsenges. Phys. Chem. 74, 476 [1970]. $r$ ist ein sogen. "virtueller“ Zwischenzustand des Moleküls. Das Integral erstreckt sich über den ganzen Konfigurationsraum $\tau$.

Befindet sich die Laserlinie $v_{0}$ in der Flanke einer Absorptionsbande, so wird in Gl. (1) der Nenner $v_{r n}-v_{0}$ sehr klein.

Die Intensität der Raman-Bande kann dann sehr groß werden. Entscheidend ist jedoch, daß im Zähler der Gl. (1) nicht die Quadrate von Übergangsmomenten stehen, sondern dyadische Produkte, die verschiedene Vorzeichen haben können. Dadurch können sich verschiedene Glieder der Summen über $r$ gegenseitig schwächen oder gar aufheben. Man spricht von "Interferenz der Wahrscheinlichkeiten“.

Die inzwischen auf dem Markt erschienenen Argon-Krypton-Ionenlaser emittierten im sichtbaren Spektralbereich so viele Laserlinien, so daß es grundsätzlich möglich ist, von sehr vielen, intensiv gefärbten Substanzen Resonanz-Raman-Spektren zu erhalten. Auf diese Weise könnte zukünftig die RamanSpektroskopie auch einen breiteren Anwendungsbereich in der analytischen Chemie finden. Im folgenden werden die Resonanz-Raman-Spektren der in Acetonitril gelösten Thiocyanatkomplexe der Übergangsmetalle Eisen, Kupfer und Kobalt sowie Rhenium und Technetium diskutiert.

\section{Experimentelles}

Die Raman-Spektren wurden mit dem Perkin-ElmerSpektrometer LR-1 aufgenommen, das von uns zur Gewährleistung ausreichender Intensitäten mit einem 50-mW-He-Ne-Laser, Modell 125, der Firma S pe c tra Physics, Mountain View, Calif., ausgerüstet wurde. Die Wellenlänge des Laserlichtes beträgt $6328 \AA$ A Zur Aufnahme der Infrarotspektren diente das Infrarotspektrometer 225 von Perkin-E lmer. Alle verwendeten Präparate hatten den Reinheitsgrad p. A.; das Acetonitril hatte die Qualität „uvasol“ E. M erck, Darmstadt.

Die Komplexierung von Eisen, Kobalt und Kupfer erfolgte durch Zugabe von Lithiumthiocyanat, wobei in allen Fällen ein Konzentrationsverhältnis von Zentralatom zum Komplexbildner $\left(\mathrm{SCN}^{-}\right)$von $1: 10$ eingestellt wurde.

Die Ionenstärke wurde mit Lithiumperchlorat auf 0,1 eingestellt.

5 J. Behringer u. J. Brandmüller, Z. Elektrochem., Ber. Bunsenges. Phys. Chem. 60, 643 [1956].

6 J. Behringer, Z. Elektrochem., Ber. Bunsenges. Phys. Chem. 62, 906 [1958]. 
Durch systematische Aufnahme von Raman-Spektren innerhalb des ausgedehnten Konzentrationsbereiches $10^{-1} \mathrm{~m}$ bis $5 \cdot 10^{-6} \mathrm{~m}$ mußte die jeweilige optimale Konzentration für den Resonanz-Raman-Effekt empirisch ermittelt werden. Die in den Abb. 1, 3, 5, 8 und 9 dargestellten Raman-Spektren sind unter näherungsweise optimalen Konzentrationsbedingungen aufgenommen, unter denen der Resonanz-Raman-Effekt voll zur Geltung kommt und die Absorption eine untergeordnete Rolle spielt. Hingegen erfolgte die Aufnahme der IR-Spektren einheitlich bei der Konzentration $10^{-2} \mathrm{~m}$.

Die Thiocyanate des Rhenium und Technetium lagen als Tetramethylammoniumsalze vor, mit den chemischen Bruttoformeln *

$\left(\mathrm{CH}_{3}\right)_{4} \mathrm{~N}\left[\mathrm{Re}(\mathrm{NCS})_{6}\right] \quad$ bzw. $\quad\left(\mathrm{CH}_{3}\right)_{4} \mathrm{~N}\left[\mathrm{Tc}(\mathrm{NCS})_{6}\right]$. Sie wurden wegen der großen Stabilität der Komplexe direkt in Acetonitril gelöst und gemessen. Eine Einstellung der Ionenstärke mittels Lithiumperchlorat unterblieb. Die Konzentrationen der Proben für die RamanMessungen lagen im Bereich von $10^{-3}$ bis $5 \cdot 10^{-6} \mathrm{Mo} / 1$; für die IR-Messungen bei $1 \cdot 10^{-2} \mathrm{Mol} / \mathrm{l}$.

\section{Resonanz-Raman-Effekt bei Thiocyanaten $\operatorname{des} \mathrm{Fe} ; \mathrm{Co} ; \mathrm{Cu}$}

\section{a) Verhalten der Komplexe}

Ein ausgeprägter Resonanz-Raman-Effekt wird bei den Thiocyanaten des Eisen, Kobalt und Kupfer in Acetonitril beobachtet. Alle drei untersuchten Übergangsmetalle bilden intensiv gefärbte Komplexe. Rot gefärbtes Eisenthiocyanat bildet den am stärksten gefärbten Komplex; braunes Kupferthiocyanat und blaues Kobaltthiocyanat besitzen bei weitem nicht die gleiche Farbintensität. Für die Stärke des Resonanz-Raman-Effektes ist nicht nur der Abstand der Absorptionsbande von der Erregerlinie maßgebend, sondern auch die Intensität der Absorptionsbande. Eisenthiocyanat und Kobaltthiocyanat haben wesentlich intensivere Absorptionsbanden als Kupferthiocyanat. Aus diesem Grunde sollten auch Eisenund Kobaltthiocyanat einen wesentlich stärkeren Resonanz-Raman-Effekt zeigen als Kupferthiocyanat. Allerdings erweisen sich die tatsächlichen Verhältnisse als ziemlich kompliziert. Einmal liegen keine reinen Thiocyanatkomplexe vor, sondern es sind zugleich ein oder mehrere Acetonitrilmoleküle als Liganden an das zentrale Metallatom des Komplexes

* Diese Komplexe wurden dankenswerterweise von Dr. K. Schwochau und Mitarbeitern, Institut für Radiochemie der KFA, hergestellt.

7 G. Wolff u. I. Büker, Z. Anal. Chem. (in Vorbereitung). gebunden. Weiterhin findet im intensiven Lichtfeld des Lasers eine teilweise Zersetzung der Komplexe statt. Während bei Kobaltthiocyanat keine Entfärbung oder Ausflockung zu beobachten ist, entfärbt sich die Lösung von Kupferthiocyanat im Verlaufe mehrerer Minuten. Daher muß beim Durchmessen des gesamte Wellenzahlbereiches die Probelösung etwa alle $50 \mathrm{cmff}^{1}$, entsprechend einer Zeit von ca. 5 min, ausgewechselt werden. Noch viel eklatanter ist die Entfärbung von Eisenthiocaynat. Innerhalb von ein bis zwei Minuten tritt bereits eine vollständige Entfärbung der Lösung im Laserlicht ein und die Lösung muß alle $10-20 \mathrm{~cm}^{-1}$, entsprechend einer Zeit von $1 / 2-1 \mathrm{~min}$, ausgewechselt werden.

Aus diesem Grunde erscheint es nicht sinnvoll, exakte Intensitätsvergleiche durchzuführen, während ein qualitativer Vergleich der Intensitäten möglich ist. Der Vollständigkeit halber und als Stütze zur Interpretation der Raman-Spektren wurden auch die Infrarotspektren der Thiocyanate aufgenommen. Es wurde außerdem versucht, durch Aufnahme von UV. Spektren die Stabilitätskonstanten der einzelnen Komplexe und aus diesen deren Koordinationszahlen und Existenzbereiche zu ermitteln. Aus Löslichkeitsgründen und wegen der starken chemischen Wechselwirkungen mit dem Solvens entstanden dabei gewisse Schwierigkeiten, so daß zur Zeit nur für das System Kobaltthiocyanat einigermaßen zuverlässige Daten gewonnen werden konnten ${ }^{7}$.

\section{b) Diskussion der Spektren}

Die Infrarot- und Raman-Spektren der untersuchten Übergangsmetallthiocyanatkomplexe sind in den Abb. 1 bis 7 wiedergegeben und die gefundenen Banden sind in Tab. 1 zusammengestellt. Zum Vergleich ist auch das Infrarotspektrum von in Acetonitril gelöstem Lithiumthiocyanat angegeben.

Mit großer Wahrscheinlichkeit liegen Komplexe des $\mathrm{Fe}$; $\mathrm{Co}$ und $\mathrm{Cu}$ mit einer gemischten Koordinationssphäre aus $\mathrm{SCN}^{-}$und Acetonitrilliganden unter unseren Arbeitsbedingungen vor ${ }^{8}$. Dies geht aus den Infrarotspektren hervor. Die Banden zwischen 2310 und $2240 \mathrm{~cm}^{-1}$ ändern für die verschiedenen Zentralionen sowohl etwas ihre Lage als auch ihre In-

8 Über Komplexe des Silbers in Acetonitril mit einer gemischten Koordinationssphäre aus $\mathrm{NO}_{3}{ }^{-}$- und Acetonitrilliganden berichten G. J. JANZ und Mitarbeiter ${ }^{9}$.

9 G. J. Janz, K. Balasubrahmanyam u. G. Oliver, Abstracts "IUPAC Symposium on Non-aqueous Electrochemistry“, Paris, 8.-10. Juni 1970. 


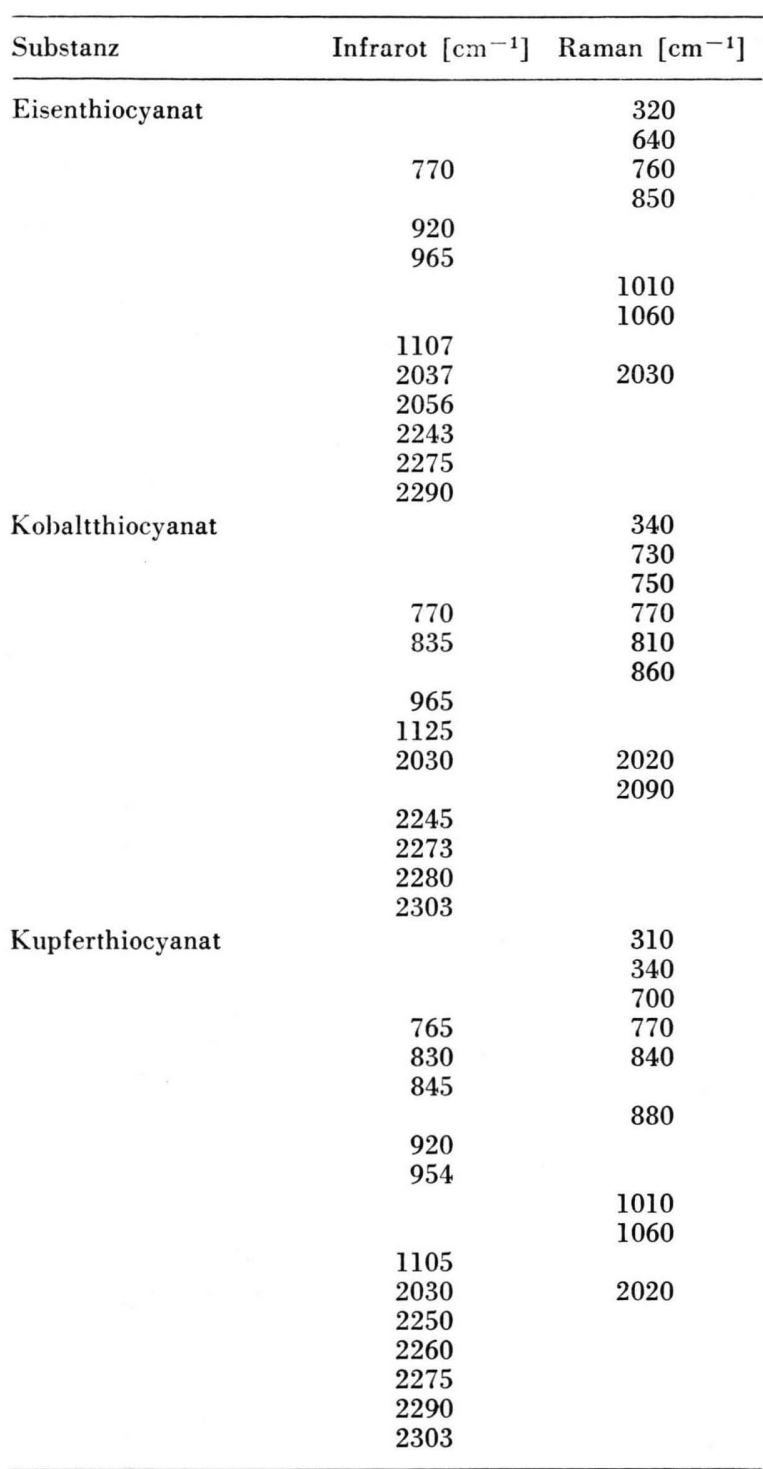

Tab. 1. Raman- und Infrarot-Daten der Thiocyanate des Eisen, Kobalt und Kupfer in Acetonitril.

tensität. Dies verdeutlicht ebenfalls eindeutig die Feinstruktur der Bande zwischen 910 und $930 \mathrm{~cm}^{-1}$, welche für die verschiedenen Zentralionen unterschiedliche Gestalt besitzt. In allen Fällen tritt im Infrarotspektrum die $\mathrm{C} \equiv \mathrm{N}$-Schwingung des freien $\mathrm{SCN}^{-}$-Ions bei $2075 \mathrm{~cm}^{-1}$ auf. Man beobachtet in

10 Beim Eisen werden zwei Banden $\left(2037 \mathrm{~cm}^{-1} ; 2056 \mathrm{~cm}^{-1}\right)$ beobachtet.

11 Die Raman-Bande bei $2090 \mathrm{~cm}^{-1}$ bei Kobaltthiocyanat dürfte einem Zersetzungsprodukt zuzuschreiben sein.

12 M. M. Chamberlain u. J. C. Bailar JR., J. Amer. Chem. Soc. 81, 6412 [1959]. der Flanke dieser Bande im Bereich von 2000 bis $2060 \mathrm{~cm}^{-1}$ jeweils noch eine weitere schwächere Bande ${ }^{10}$. Dies muß die $\mathrm{C} \equiv \mathrm{N}$-Valenzschwingung des komplexgebundenen Thiocyanations sein. Im Raman-Spektrum tritt ebenfalls in diesem Bereich in allen Fällen die analoge Bande bei $2020 \mathrm{~cm}^{-1}$ bzw. $2030 \mathrm{~cm}^{-1}$ auf ${ }^{11}$. Die im IR bei großen Wellenzahlen zwischen 2310 und $2240 \mathrm{~cm}^{-1}$ auftretenden Banden konnten im Raman-Spektrum nicht registriert werden, da keine Möglichkeit zur Kompensation der Acetonitrilbanden bestand. Die Banden zwischen 1100 und $1130 \mathrm{~cm}^{-1} \mathrm{im}$ Infrarotspektrum müssen Schwingungen von komplexgebundenem Acetonitril zugeordnet werden. Sie konnten im Raman-Spektrum nicht eindeutig nachgewiesen werden. Mit relativ größter Intensität tritt im Resonanz-RamanSpektrum die Bande im Bereich zwischen 740 und $780 \mathrm{~cm}^{-1}$ auf. Sie wird der totalsymmetrischen S - C. Valenzschwingung des komplexgebundenen Thiocyanations zugeordnet. Diese Bande tritt ebenfalls im Infrarotspektrum an der gleichen Stelle auf. Die bis jetzt bekannten $\mathrm{S}-\mathrm{C}$-Schwingungen der Thiocyanate von $\mathrm{Fe}$ und Co liegen im Infrarot ${ }^{12-16}$ zwischen 820 und $750 \mathrm{~cm}^{-1}$. Diese Messungen erfolgten an reinen Thiocyanatkomplexen. Durch die zusätzliche Anlagerung von Acetonitril kann die Bindung der S-C-Gruppierung etwas schwächer werden, so daß die vorgenommene Zuordnung sinnvoll erscheint. Eine andere Möglichkeit wäre, die Schwingungsbande zwischen 800 und $900 \mathrm{~cm}^{-1}$ der S-CValenzschwingung zuzuordnen. Allerdings läge dann die S-C-Schwingung ziemlich kurzwellig und wäre energetisch höher als die S-C-Valenzschwingung in reinen Thiocyanatkomplexen. Die Bande bei 800 bis $900 \mathrm{~cm}^{-1}$ ist also entweder eine Schwingung des komplexgebundenen Acetonitrils oder eine Bande eines Zersetzungsprodukts.

Bei kleineren Wellenzahlen zwischen 300 und $350 \mathrm{~cm}^{-1}$ findet man noch schwache Banden im Raman-Spektrum. Hierbei handelt es sich möglicherweise um die ins Längerwellige verschobene Bande, die bei $380 \mathrm{~cm}^{-1}$ für das freie Acetonitrilmolekül auftritt. Eine andere Möglichkeit wäre, diese Bande der Metall-Schwefel- bzw. Metall-Stickstoffschwingung zuzuordnen.

13 J. Lewis, R. S. Nyholm u. P. W. Smith, J. Chem. Soc. $1961,4590$.

14 A. Tramer, J. Chim. Phys. 59, 232 [1962].

15 A. Turco u. C. Pecile, Nature London 191, 66 [1961].

16 A. Wojcicki u. M. F. Farona, Inorg. Chem. 3, 151 [1964]; 4, 857 [1965]. 


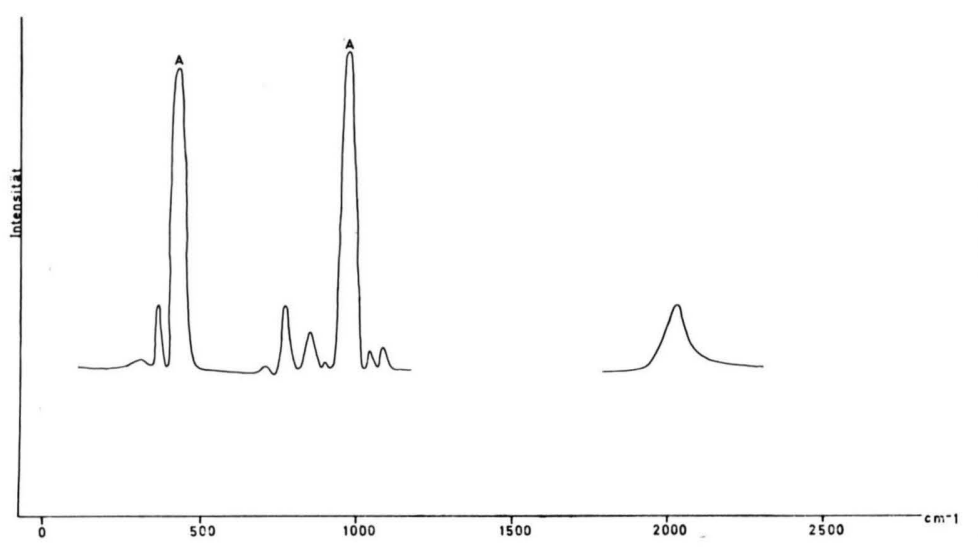

Abb. 5. Raman-Spektrum von Kupferthiocyanat in Acetonitril (Konzentration bezogen auf $\mathrm{CuCl}_{2}: 1 \cdot 10^{-3} \mathrm{~mol} / \mathrm{Liter}$ ); $\mathrm{A}=$ Banden des Acetonitril.

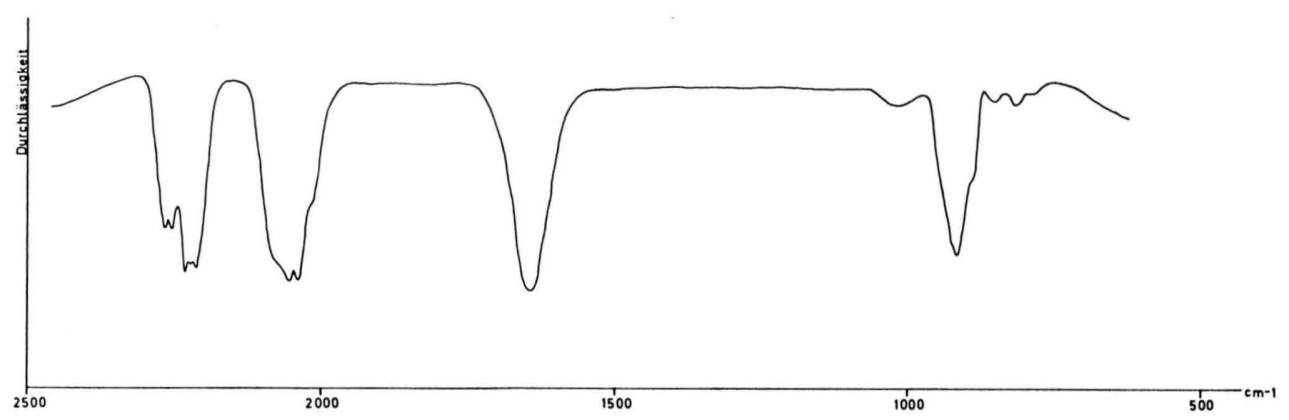

Abb. 6. IR-Spektrum von Kupferthiocyanat in Acetonitril

(Konzentration bezogen auf $\mathrm{CuCl}_{2}: 1 \cdot 10^{-2} \mathrm{~mol} /$ Liter)

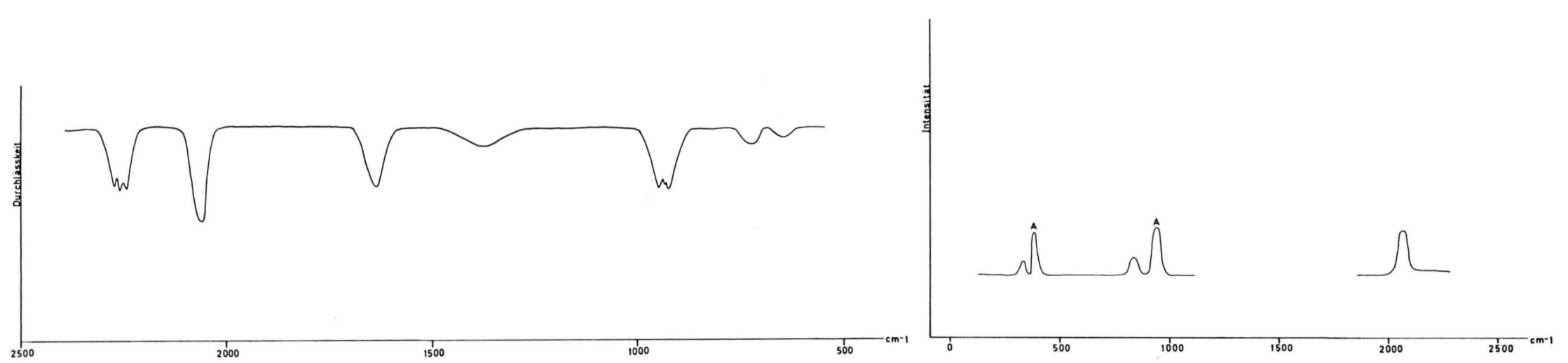

Abb. 7. Infrarotspektrum von Lithiumthiocyanat in Acetonitril $\left(1 \cdot 10^{-2} \mathrm{~mol} /\right.$ Liter $)$.

Abb. 8. Raman-Spektrum von Technetiumthiocyanat in Acetonitril; $\mathrm{A}=$ Banden des Acetonitril. 


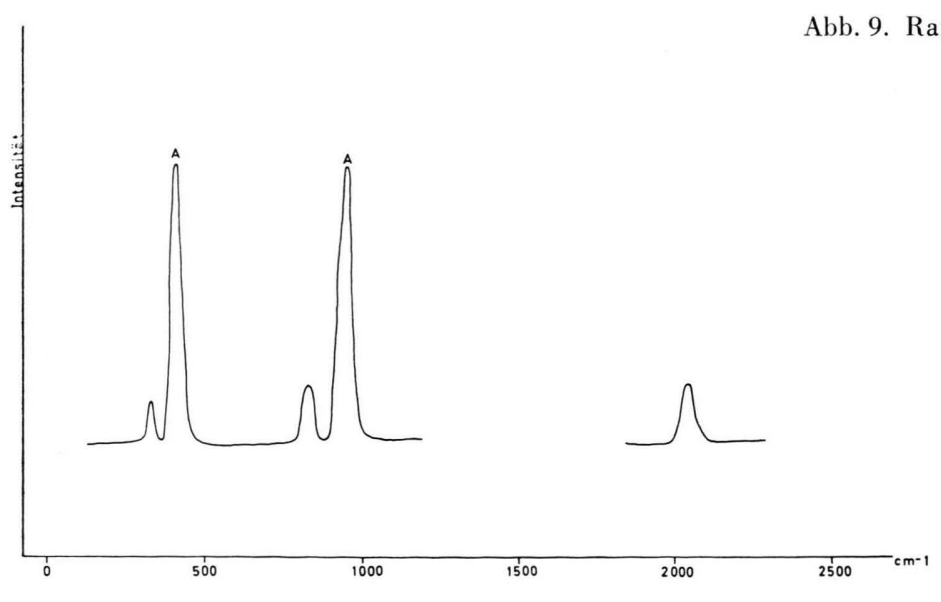

aman-Spektrum von Rheniumthiocyanat in Acetonitril; $\mathrm{A}=$ Banden des Acetonitril.

Es hat sich gezeigt, daß die $\mathrm{C} \equiv \mathrm{N}$-Valenzschwingung des komplexgebundenen Thiocyanats sowohl im Raman- als auch im IR-Spektrum erlaubt ist. Das gleiche gilt für die $\mathrm{S}-\mathrm{C}-$ Valenzschwingung. Aus diesem Grunde kann gefolgert werden, daß das Alternativverbot nicht gilt und kein hochsymmetrischer Komplex mit einem Symmetriezentrum vorliegt. Die Symmetrieerniedrigung wird durch die Anlagerung eines oder mehrerer Acetonitrilmoleküle hervorgerufen. Weiterhin besteht die Möglichkeit, daß die Intensität im Raman-Spektrum erst durch die Anlagerung von Acetonitrilmolekülen induziert wird.

Die Intensität im Raman-Spektrum hängt generell stark von der Umgebung der streuenden Moleküle und damit von den zwischenmolekularen Kräften ab. Insbesondere spielen Molekülassoziationen und Anlagerung von Lösungsmittelmolekülen eine entscheidende Rolle. Je nach dem Lösungsmittel hat eine Absorptionsbande eine verschobene Lage und Struktur ${ }^{5}$. Eine geringfügige Verschiebung kann jedoch bereits eine sehr große Intensitätsänderung der Raman-Linien verursachen.

Auch in Methanol wurde ein ausgeprägter Resonanz-Raman-Effekt bei den Thiocyanaten von Fe; $\mathrm{Co}$ und $\mathrm{Cu}$ beobachtet. Hingegen war in Dimethylsulfoxyd nur noch bei den Thiocyanaten von $\mathrm{Fe}$ und Co eine gewisse Verstärkung der Intensitäten der Raman-Banden wahrnehmbar, während bei Cu bereits ein nahezu normales Verhalten der RamanIntensität auftrat ${ }^{3}$.

17 H. Siebert, Anwendungen der Schwingungsspektroskopie in der anorganischen Chemie, Springer-Verlag, Berlin 1966.

\section{Resonanz-Raman-Effekt an Thiocyanaten des Technetiums und Rheniums}

Die Thiocyanate des Rheniums und Technetiums wurden ebenfalls in Acetonitril untersucht. Während jedoch braunes Rheniumthiocyanat relativ beständig im Lichtfeld des Lasers ist und sich mit der Zeit kaum entfärbt, tritt bei violettem Technetiumthiocyanat eine sehr rasche Entfärbung und Ausflockung ein, so daß das Raman-Spektrum stückweise mit jeweils frischen Probelösungen aufgenommen werden mußte. Die entsprechenden Raman-Spektren sind in den folgenden Abb. 8 und 9 dargestellt und die gefundenen Banden in Tab. 2 angegeben. Wegen der Unbeständigkeit der untersuchten Thiocyanate und um generell das Verhalten in fester Form und gelöster Phase zu vergleichen, wurden auch die Spektren in kristallinem Zustand aufgenommen.

\begin{tabular}{|c|c|c|}
\hline Substanz & $\begin{array}{l}\text { Raman-Spektrum } \\
\text { in Kristall } \\
\mathrm{cm}^{-1}\end{array}$ & $\begin{array}{l}\text { Raman-Spektrum } \\
\text { in } \mathrm{CH}_{3} \mathrm{CN} \\
\mathrm{cm}^{-1}\end{array}$ \\
\hline \multirow{3}{*}{$\left(\mathrm{CH}_{3}\right)_{4} \mathrm{~N}\left[\operatorname{Re}(\mathrm{NCS})_{6}\right]$} & $320 ?$ & $325 ?$ \\
\hline & 820 & 815 \\
\hline & 2065 & 2060 \\
\hline \multirow[t]{3}{*}{$\left(\mathrm{CH}_{3}\right)_{4} \mathrm{~N}\left[\mathrm{Tc}(\mathrm{NCS})_{6}\right]$} & $340 ?$ & $433 ?$ \\
\hline & 830 & 826 \\
\hline & 2070 & 2070 \\
\hline
\end{tabular}

Tab. 2. Raman-Banden der Thiocyanate des Re(IV) und Tc (IV).

In gelöstem Zustand existieren Komplexe der Formel $\left[\operatorname{Re}(\mathrm{NCS})_{6}\right]^{-}$bzw. $\left[\mathrm{Tc}(\mathrm{NCS})^{6}\right]^{-}$. Aus der Tatsache, daß bei analogen Thiocyanatkomplexen $\left[\mathrm{Me}(\mathrm{NCS})_{6}\right]^{-}$eine oktaedrische Struktur vorliegt ${ }^{17}$ und auf Grund von Infrarotdaten der Technetium- 